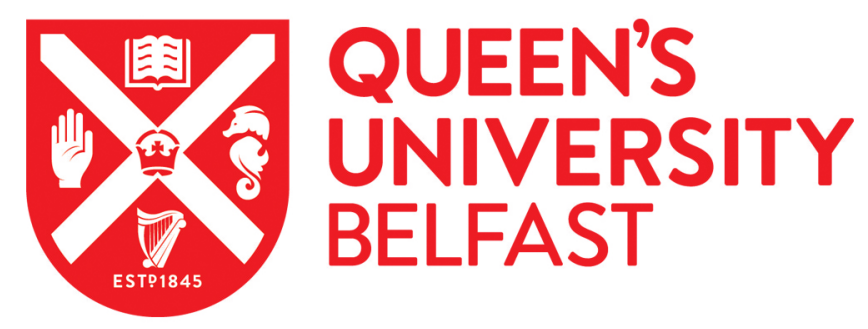

\title{
Impact of prosthodontic rehabilitation on the masticatory performance of partially dentate older patients: can it predict nutritional state?
} Results from a RCT

Wallace, S., Samietz, S., Abbas, M., McKenna, G., Woodside, J. V., \& Schimmel, M. (2018). Impact of prosthodontic rehabilitation on the masticatory performance of partially dentate older patients: can it predict nutritional state? Results from a RCT. Journal of Dentistry, 68, 66-71. https://doi.org/10.1016/j.jdent.2017.11.003

Published in:

Journal of Dentistry

Document Version:

Peer reviewed version

Queen's University Belfast - Research Portal:

Link to publication record in Queen's University Belfast Research Portal

\begin{abstract}
Publisher rights
(c) 2017 Elsevier.

This manuscript is distributed under a Creative Commons Attribution-NonCommercial-NoDerivs License

(https://creativecommons.org/licenses/by-nc-nd/4.0/), which permits distribution and reproduction for non-commercial purposes, provided the author and source are cited.
\end{abstract}

\section{General rights}

Copyright for the publications made accessible via the Queen's University Belfast Research Portal is retained by the author(s) and / or other copyright owners and it is a condition of accessing these publications that users recognise and abide by the legal requirements associated with these rights.

\section{Take down policy}

The Research Portal is Queen's institutional repository that provides access to Queen's research output. Every effort has been made to ensure that content in the Research Portal does not infringe any person's rights, or applicable UK laws. If you discover content in the

Research Portal that you believe breaches copyright or violates any law, please contact openaccess@qub.ac.uk. 


\section{Abstract (250/ 250 words)}

Impact of prosthodontic rehabilitation on the masticatory performance of partially dentate older patients: can it predict nutritional status? Results from a RCT.

Objectives: With a decreased number of teeth, a reduction in chewing function can contribute to changes in food choices and ultimately impact on overall nutritional status. This study compared the impact of two tooth replacement strategies for partially dentate older patients on masticatory performance and nutritional status.

Methods: Patients aged 65 years and older were randomly allocated to two different treatment groups. For the RPDP-group (removable partial dental prostheses) each participant was restored to complete arches with cobalt-chromium removable prostheses to replace missing teeth. For the SDA-group (shortened dental arch), participants were restored to 10 occluding pairs of natural and replacement teeth using adhesive bridgework. Masticatory performance was assessed with a colour-mixing ability test. Each patient provided haematological samples that were screened for biochemical markers of nutritional status. Patients were also assessed using the Mini Nutritional Assessment (MNA).

Results: Eighty-nine patients completed the test for masticatory performance and provided blood samples and MNA scores at baseline (BL) and after 12 months (12m). Masticatory performance $(\mathrm{p}<0.001)$ and MNA $(\mathrm{p}<0.05)$ increased significantly in both groups, but no significant between group differences were noted. A mixed picture was observed for nutrition biomarkers. Mixed-effect linear regression models did not demonstrate that nutritional status could be predicted from masticatory performance. Conclusions: These results indicate that prosthodontic rehabilitation according to the principles of the SDA is equivalent to RPDPs in terms of restoration of chewing capacity 
for partially dentate older patients. However, masticatory performance may only have minor associations with nutritional status for this patient group.

Clinical significance: (32/ 50 words) Replacing teeth with either RPDPs or SDA provides a prerequisite for efficient chewing. Further research is required to determine the impact of oral rehabilitation coupled with nutritional counselling for this patient population. 


\section{Introduction}

As preventative strategies continue to advance, more and more older adults are now retaining natural teeth into old age. This trend can be seen throughout most of the western world and has resulted in an increasing proportion of partially dentate older patents. Consequently, the prevalence of edentulism is becoming an increasingly scarce condition in high-income households in countries such as North America [1]. Projections suggest that in future, edentulousness will be seen almost exclusively within the older, economically deprived population. Whilst natural tooth retention is a very positive development, natural teeth are at significant risk from chronic dental diseases particularly when coupled with xerstomia [2]. Age-related systemic diseases can make it extremely difficult to clean remaining natural teeth and removable dental prostheses, therefore the maintenance burden is very high. In a review, Gonda et al. state that removable partial dental prostheses (RPDP) may be a significant factor for further multiple tooth loss and have the potential to contribute to oral disease [3].

In older people, it has been shown that the perceived ability to chew foods is closely associated with the number of natural teeth remaining, as well as the number of occluding pairs of teeth $[4,5]$. There are many factors that can potentially influence food choice, such as socio-economic status, cultural beliefs and personal preference. However the physical ability to bite and chew is also very important [6]. There is evidence to suggest that a reduction in the number of natural teeth can subsequently reduce chewing ability, with edentate patients demonstrating poorer chewing ability compared to patients who are dentate [7]. This has been shown to result in changes in food choice behaviour, as well as a decreased enjoyment of food [7]. Further, a reduced, unrestored dentition is associated with increased mortality risk [8]. When fewer natural teeth are present, older adults tend to develop food choice habits based around foods that are softer and easier to chew. Further, for example, serum 25-hydroxyvitamin D 
for tooth loss [9]. However, these foods are often low in nutrients and fibre but high in calories and complex carbohydrates $[10,11]$. Some of the possible reasons for this are thought to be difficulty in chewing hard foods such as certain raw vegetables and fruits, and a diminished sense of taste.

Chewing is a complex function of the oro-facial system. The ultimate goal is to break down and lubricate foodstuffs, and thus form a cohesive bolus that is safe to swallow [12]. This process involves numerous structures including muscles, mucosa, salivary glands, central and peripheral nerves as well as cortical and basal structures and receptors [13] [14]. However, the most important predictor for masticatory function is the number and distribution of natural teeth and dental prostheses [15]. Loss of occluding tooth pairs can result in reduced chewing efficiency and may in turn influence nutritional status. [16]. Chewing efficiency with a reduced dentition may result in a reduction in masticatory performance by up to $40 \%$ and RPDPs may only partially compensate this functional deficit [17] [18]. Prosthodontic rehabilitation has previously been demonstrated to provide mixed results in improving masticatory ability in partially dentate older patients [19].

The aim of this study was to compare the chewing efficiency of partially dentate older patients with RPDP with those restored to a SDA. The study also aimed to investigate if an improvement in masticatory function was associated with an improvement in overall nutritional status.

\section{Material and Methods}

\subsection{Study Design}

A randomised controlled clinical trial (RCT) was conducted to compare the two treatment modalities (Trial Registration no. ISRCTN26302774). The inclusion criteria for patients involved in the study were: aged 65 years and older; minimum of six remaining sound natural teeth in either arch, seeking replacement for missing natural 
teeth; ability to accept routine dental care in a dental chair; no medical conditions which could prevent routine dental treatment. Two centres were used for patient recruitment; Cork University Dental Hospital (CUDH) and St Finbarr's Geriatric Day Hospital (SFDH) in Cork, Ireland. Patients recruited from SFDH represented an older and more systemically unwell cohort. Before treatment commenced, written information regarding the proposed treatment was provided for each patient involved in the study, and each patient also completed a written consent form prior to treatment. The Cork Teaching Hospitals Ethics Committee granted full ethical approval for the study (ref: ECM 5 (9) 05/02/08).

\subsection{Randomisation}

In this study patients were randomly allocated to one of two treatment groups; the RPDP treatment group, or the SDA treatment group. A computer-generated schedule in SAS@ was utilised to perform the randomization remote from the clinical setting. The randomisation was stratified according to gender and age, and was carried out in blocks of varying length. For both the CUDH and SFDH recruitment sites a separate schedule for the randomisation was produced. Both the RPDP and SDA treatment groups contained patients recruited from both recruitment sites, and these were randomised independently. The randomisation procedure was performed by a research assistant, and the allocation of treatment groups was carried out by the clinical operator who was blinded to the allocation. For the duration of the study patients were assessed using an intention to treat analysis; patients were analysed according to the initial treatment intent (determined by the randomisation process), and not the treatment eventually administered.

\subsection{Operative care}

For the patients to be involved in the study they were required to be dentally fit. Prior to treatment onset they received standardised dental care which included extraction of 
unrestorable teeth, restoration of caries and replacement of defective direct

restorations, extensive oral hygiene instruction and non-surgical periodontal treatment. The patients within the RPDP group had complete arch restoration; with missing teeth replaced using cobalt-chromium frameworks. For the SDA group patients, adhesive resin bonded bridgework (RBB) was carried out according to a standardised protocol throughout the arch to restore patients to a premolar occlusion of 10 occluding pairs of natural and replacement teeth. Posterior teeth distal to the SDA were left unopposed. Minimal tooth preparation within enamel only was carried out to produce retentive forms and increase the surface area for bonding. For the purpose of this study the RPDPS and RBB used were constructed by the same dental laboratory and all operative treatment was conducted by a single clinician with postgraduate training in prosthodontics.

\subsection{Data collection}

The primary outcome measure for this RCT was oral health related quality of life (OHRQoL), which was measured using OHIP-14 [20]. Masticatory performance was included as a secondary outcome measure.

\subsubsection{Masticatory performance.}

Masticatory performance was assessed with a previously validated colour-mixing ability, i.e. bolus-kneading, test [21, 22]. A two-coloured chewing gum (Vivident Fruitswing “Karpuz/ Asai Üzümü”, Perfetti van Melle, Turkey) was used. The gum contained a green and dark violet layer with the dimensions $43 \times 12 \times 3 \mathrm{~mm}$. The gum was relatively soft (Shore hardness 00: green side: mean depth of indentation $0.8 \pm 0.08 \mathrm{~mm}$, mean Durometer 67.4, violet side: mean depth of indentation $1.1 \pm 0.08 \mathrm{~mm}$, mean Durometer 58.4) and did not adhere to denture resin, artificial teeth or to cast metal frameworks. 
The chewing gum was placed in each patient's mouth and they were asked to chew normally, with the operator counting the chewing strokes. After 20 chewing cycles, the gum was retrieved from the oral cavity, dried and placed in a transparent plastic bag. The gum was then flattened to a $1 \mathrm{~mm}$ thick wafer. Both sides of the wafer were scanned with a standard flatbed scanner (Epson Perfection V37, Espon Deutschland GmbH, Kloten, Switzerland) at a resolution of $300 \mathrm{dpi}$ and saved in *jpg format. Both images were then jointly imported into the ViewGum (c) (www.dhal.com) software. The software transformed the images in the HIS (hue, intensity, saturation) colour space and separated semi-automatically the gum from the background. The hue component in this colour space showed two well-separated peaks, when the colours were not mixed. Those peaks gradually merged as the mixing of the colours increased. The variance of hue $(\mathrm{VOH})$ was therefore considered as the measure of mixing [23]. $\mathrm{VOH}$ is significantly associated with the number of chewing cycles and correlates to the comminution test [22]. It was therefore used as the measure for masticatory performance in this boluskneading test.

\subsubsection{Nutritional status}

To assess nutritional status, the mini nutritional assessment (MNA) was administered by a research nurse at baseline (pre-treatment) and 12 months post-treatment intervention. The research nurse was blinded to the treatment group allocation of all participants involved. Haematological samples were provided by participants and screened in Cork University Hospital for biochemical markers of nutritional status including serum albumin, serum cholesterol, ferritin, folate, vitamin B12 and 25hydroxycholecalciferol (vitamin D) according to standard hospital protocols. Haematological samples were collected from each participant at baseline and 12 months post-treatment intervention, by a research nurse blinded to the treatment group allocation. 


\subsection{Sample size calculation}

A power calculation was made based on summary OHIP-14 score data from the UK Adult Dental Health Survey as this was the primary outcome measure for the study. The sample size calculation $(\alpha=0.05, \beta=0.8)$ indicated that 44 patients from both treatment groups were required to complete the study, however the aim was to overrecruit patients to allow for a drop-out rate of up to $30 \%$. The sample size calculation was also checked to ensure validity for masticatory performance as a secondary outcome measure. The null hypothesis tested was that there would be no difference observed in masticatory performance between the RPDP Group and the SDA Group. Data from a previous study employing the same methodology was used [22]. The effect size d was 1.77 for VOH between the investigated groups and it was calculated that a total sample size of 40 ( $n=20$ in each group) was necessary to achieve sufficient power $(\alpha=0.0001, \beta=0.90)$ to reject the null-hypothesis. The calculation was performed using PS Power and Sample Size Calculations Version 3.1 [24].

\subsection{Statistical Analysis}

All variables recorded were summarised using appropriate descriptive statistics and graphics, and presented by time-point and treatment group. All statistical analysis was carried out using R, version 3.2.2 (The R Project for Statistical Computing, Vienna, Austria), with the additional package "lme4". Frequency and descriptive statistics were calculated for numerical and categorical variables (mean, standard deviation, median and quantiles as appropriate). Student's t tests were performed to compare means and frequencies were compared using a binomial test for proportions. In order to detect associations between two quantitative variables with a normal distribution, the two variables in question were plotted against each other and a Pearson's correlation carried out, to indicate the strength and direction of the association between the two variables. Masticatory performance (VOH) was also further analysed with linear 
regression; with several models being performed to find the optimal predictive structure. Linear mixed effects models were also performed for flexibility for both fixed and random effects and to allow predictors to be controlled for age or gender.

For all analyses performed the level of significance was set to 0.05 .

\section{Results}

\section{Characteristics of the study participants}

A total of 89 participants were recruited into the study and randomly allocated to RPDP group ( $n=44)$ and the SDA group ( $n=45)$, respectively (Fig. 1). At baseline there were no significant differences observed between the two patient groups, with regards to gender, age, number of teeth and number of occluding units (OU). Twelve months after treatment intervention, the RPDP group had a significantly higher number of OU (Tab.

1) compared to the SDA group $(\mathrm{p}<0.0001)$.

\section{Masticatory performance}

Masticatory performance was very similar at baseline for both groups and increased significantly 12 months after prosthodontic rehabilitation (differences for both groups $\mathrm{p}<0.001)$. However, there were no significant differences between groups observed $(\mathrm{p}=0.169)$ (Fig. 2).

\section{Nutritional parameters}

A detailed article on the nutritional parameters has been reported elsewhere [25]. A mixed-model analysis of covariance (ANCOVA) was performed to investigate haematological markers for both the RPDP and SDA treatment groups. When the two treatment groups were compared, no statistically significant differences were observed after 12 months for the following markers; Vitamin B12 ( $p=0.94)$, Serum Folate ( $p=$ 0.58), Ferritin $(p=0.70)$, Albumin $(p=0.82)$, Serum Total Cholesterol $(p=0.37)$ and 
Vitamin D ( $\mathrm{p}=0.7666)$. Whilst MNA scores showed some improvements over the length of the study $(p<0.05)$ these were similar within the two treatment groups $(p>0.05)$.

\section{Predictors of nutritional state}

As baseline and at the 12 months follow-up, no significant correlations were observed between masticatory performance ( $\mathrm{VOH})$ and MNA score or any of the haematological biomarkers tested (Tab 2). The mixed-effects linear regression model illustrated that during the course of this study, MNA score increased at each time point after treatment intervention. Increasing age was significantly associated with lower MNA scores and therefore with diminished nutritional state. Applying the same statistical model, Vitamin D levels increased during the study period and could be predicted from the assessment time point. All of the other potentially predictive factors were not statistically significant in the applied regression model (Tab. 3).

\section{Discussion}

\section{Principal findings}

In summary, this RCT demonstrates a significant improvement in masticatory function in partially dentate older adults rehabilitated with either RPDPs or according to the principles of the SDA. Although the number of occluding antagonist pairs of teeth was significantly higher in patients with RPDP, there was no significant difference in the increase in masticatory function compared to patients restored to a SDA. The study results illustrate that nutritional status is not consistently associated with masticatory function based on linear regression models. However, with increasing masticatory function some nutritional parameters, including MNA, improved. 


\section{Critique of the method}

\section{Chewing assessment}

Chewing may be assessed subjectively, i.e. chewing ability or objectively. For the objective evaluation numerous methods have been described [26]. These comprise comminution tests, bolus-mixing ability tests and shearing capacity tests. The gold standard for assessing chewing efficiency remains today the sieving test in which a breakable foodstuff is chewed for a given number of chewing cycles or until the swallowing reflex begins [27]. The test food is spat out, cleaned, dried and analyzed according to the Rosin-Rammler formula. This method is very precise, but depends largely on the maximum available biting force. It is time consuming, messy and necessitates a laboratory setting. Furthermore, the particles produced may comprise a choking risk in elderly or dysphagic patients. Therefore, bolus-kneading tests have been developed, which are simply to use, cost-effective and quick to administer. The test specimens usually comprise coloured waxes or chewing gum, which form a cohesive bolus which is easy to control, even in stroke patients $[21,28]$. Comminution and boluskneading tests are positively and significantly correlated [22].

\section{Chewing assessment results}

The current randomized controlled clinical trial was performed with an adequate sample size, both for the primary and secondary outcome measures utilised. The number of teeth and number of occluding posterior teeth have been described as one of the most important predictors for masticatory function $[15,29,30]$. However, this does not appear to hold true for partially dentate patients with RPDPs, especially in patients with bilateral free-end saddles where chewing and denture stability remains difficult. These findings correlate with previous clinical trial data which suggested that patients with SDA treatment had adequate masticatory function in the long term [5, 31-33] as 
well as providing satisfactory comfort and appearance [31]. Studies have also demonstrated that treatment according to the SDA concept may be a more appropriate treatment option for elderly patients, who may feel more comfortable chewing with this treatment option $[25,33,34]$. This study further demonstrates that various teethrelated factors (numbers of remaining natural teeth and numbers of occluding pairs of teeth at baseline and 12 months, controlled for age and gender), are not significant predictors of masticatory performance. This conflicts with previous research, potentially due to small sample size, which shows an association between oral health status and chewing ability [35] which would suggest the need for further research into the masticatory performance of this population group. It must be recognised that all patients in this study received standardised oral care to render them dentally fit prior to any prosthodontic interventions. This may have positively influenced chewing ability even without replacing missing units. This data was not captured as part of this study but may be interest to future work.

Removable partial dentures are a popular tooth replacement treatment offered to many patients [36]; however, there are several limitations associated with their usage in partially dentate older patients. These include high levels of non-compliance with usage [37] mainly due to impaired aesthetics or oral discomfort [38] and also an increased risk of dental disease such as caries and periodontitis [39]. Many older patients receive RPDPs as part of publicly funded dental care; and it is now an emerging issue of debate as to whether RPDP treatment is an efficient use of public funds and whether it is the most effective form of treatment for the patients concerned [38].

There are several available alternatives to RPDP treatment, including functionally orientated approaches such as the shortened dental arch (SDA) concept [40]. This strategy focuses on the anterior and premolar teeth, considered necessary for 
masticatory function and aesthetic purposes [41] and aims to achieve an acceptable level of oral function through the provision of a functional dentition of 10 occluding pairs of teeth [42]. This form of treatment has been shown to be easily maintained by patients and acceptable to clinicians, however evidence suggests that SDA treatment is an underutilised approach, but may be contraindicated in some instances [33, 43].

\section{Nutritional assessment}

Although masticatory performance at baseline and 12 months was not significantly associated with various markers of nutritional state or MNA score, it is still worth noting the magnitude of evidence suggesting the relationship between oral health status and diet and subsequently nutritional state in the older adult population [6, 7]. It is a particularly important area as it has been observed that older adults with oral health issues often avoid several foods and are at risk of nutritional deficiencies $[6,44]$. Some studies have shown that patients with missing natural teeth replaced with removable dentures can improve their dietary choices whilst other studies have shown little change [45]. Food choice and diet are influenced by a number of factors including socio-economic status and educational attainment in addition to dental status.

In this study, regression models were used to predict masticatory performance at two different time points (baseline and 12 months) and the difference between the two, using factors, including markers of nutritional status, that were also assessed at baseline and 12 months. Although none of the factors tested were significant predictors of masticatory performance, potentially due to poor model fit or small sample size, there are conflicting opinions regarding the relationship between masticatory performance, oral health status and various markers of nutritional status $[35,46]$, highlighting the need for further research in the area. 


\section{Conclusions}

These results indicate that prosthodontic rehabilitation according to the principles of the SDA is equivalent to RPDPs in terms of restoration of chewing capacity for partially dentate older patients. Enhanced masticatory performance alone does not signify improved nutritional state.

\section{Figure legends}

Figure 1: Patient flow diagram

Figure 2: Boxplot for the chewing efficiency as assessed for the Variance of Hue (VOH) at baseline and 12-months follow-up. A low VOH signifies a high chewing efficiency and vice versa. 


\section{References}

[1] G.D. Slade, A.A. Akinkugbe, A.E. Sanders, Projections of U.S. Edentulism prevalence following 5 decades of decline, Journal of dental research 93(10) (2014) 959-65.

[2] C. Stock, H. Jurges, J. Shen, K. Bozorgmehr, S. Listl, A comparison of tooth retention and replacement across 15 countries in the over-50s, Community dentistry and oral epidemiology (2015) Article first published online: 28 DEC 2015, DOI: $10.1111 /$ cdoe.12209.

[3] T. Gonda, T.C. Yang, Y. Maeda, Five-year multicenter study of magnetic attachments used for natural overdenture abutments, Journal of oral rehabilitation 40(4) (2013) 258-262.

[4] A.S. Papas, C.A. Palmer, M.C. Rounds, J. Herman, R.B. McGandy, S.C. Hartz, R.M. Russell, P. Depaola, Longitudinal Relationships between Nutrition and Oral Health, Annals of the New York Academy of Sciences 561(1) (1989) 124-142. [5] J.L. Leake, An Index of Chewing Ability, Journal of Public Health Dentistry 50(4) (1990) 262-267.

[6] A. Sheiham, J.G. Steele, W. Marcenes, S. Finch, A.W. Walls, The impact of oral health on stated ability to eat certain foods; findings from the National Diet and Nutrition Survey of Older People in Great Britain, Gerodontology 16(1) (1999) 11-20.

[7] G. Boretti, M. Bickel, A.H. Geering, A review of masticatory ability and efficiency, The Journal of prosthetic dentistry 74(4) (1995) 400-3.

[8] C. Schwahn, I. Polzer, R. Haring, M. Dorr, H. Wallaschofski, T. Kocher, T. Mundt, B. Holtfreter, S. Samietz, H. Volzke, R. Biffar, Missing, unreplaced teeth and risk of all-cause and cardiovascular mortality, Int J Cardiol 167(4) (2013) 1430-7.

[9] Y. Zhan, S. Samietz, B. Holtfreter, A. Hannemann, P. Meisel, M. Nauck, H. Volzke, H. Wallaschofski, T. Dietrich, T. Kocher, Prospective Study of Serum 25hydroxy Vitamin D and Tooth Loss, Journal of dental research 93(7) (2014) 63944.

[10] P.J. Moynihan, S. Snow, N.J. Jepson, T.J. Butler, Intake of non-starch polysaccharide (dietary fibre) in edentulous and dentate persons: an observational study, British dental journal 177(7) (1994) 243-7.

[11] P.J. Moynihan, C.E. Mulvaney, A.J. Adamson, C. Seal, N. Steen, J.C. Mathers, F.V. Zohouri, The nutrition knowledge of older adults living in sheltered housing accommodation., Journal of Human Nutrition and Dietetics 20(5) (2007) 446458.

[12] A. Woda, M. Hennequin, M.A. Peyron, Mastication in humans: finding a rationale, Journal of oral rehabilitation 38(10) (2011) 781-4.

[13] M. Schimmel, G. Voegeli, E. Duvernay, B. Leemann, F. Muller, Oral tactile sensitivity and masticatory performance are impaired in stroke patients, Journal of oral rehabilitation 44(3) (2017) 163-177.

[14] P. Haggard, L. de Boer, Oral somatosensory awareness, Neurosci Biobehav Rev 47 (2014) 469-84.

[15] K. Ikebe, K. Morii, The relationship between oral function and Body Mass Index among independently living older Japanese people, British dental journal 202(5) (2007) 275-275. 
[16] A. Sheiham, J.G. Steele, W. Marcenes, C. Lowe, S. Finch, C.J. Bates, A. Prentice, A.W. Walls, The relationship among dental status, nutrient intake, and nutritional status in older people, Journal of dental research 80(2) (2001) 408-13.

[17] C. Moore, G. McKenna, In patients with shortened dental arches do removable dental prostheses improve masticatory performance?, Evid-based Dent 17(4) (2016) 114-114.

[18] M. Schimmel, K. Memedi, T. Parga, J. Katsoulis, F. Müller, Masticatory Performance, Maximum Bite- and Lip Force Depend on the Type of Prostheses, The International journal of prosthodontics (accepted).

[19] B. Wostmann, K. Michel, B. Brinkert, A. Melchheier-Weskott, P. Rehmann, M. Balkenhol, Influence of denture improvement on the nutritional status and quality of life of geriatric patients, J Dent 36(10) (2008) 816-21.

[20] G. McKenna, P.F. Allen, D. O'Mahony, M. Cronin, C. DaMata, N. Woods, The impact of rehabilitation using removable partial dentures and functionally orientated treatment on oral health-related quality of life: a randomised controlled clinical trial, Journal of dentistry 43(1) (2015) 66-71.

[21] M. Schimmel, P. Christou, H. Miyazaki, D. Halazonetis, F.R. Herrmann, F. Müller, A novel colourimetric technique to assess chewing function using twocoloured specimens: Validation and application, Journal of Dentistry 43(8) (2015) 955-964.

[22] M.S. Kaya, B. Guclu, M. Schimmel, S. Akyuz, Two-colour chewing gum mixing ability test for evaluating masticatory performance in children with mixed dentition: validity and reliability study, Journal of oral rehabilitation (2017). [23] D.J. Halazonetis, M. Schimmel, G.S. Antonarakis, P. Christou, Novel software for quantitative evaluation and graphical representation of masticatory efficiency, Journal of oral rehabilitation 40(5) (2013) 329-35.

[24] W.D. Dupont, W.D.J. Plummer, PS Power and Sample Size Calculations Version 3.0.

<http://biostat.mc.vanderbilt.edu/twiki/bin/view/Main/PowerSampleSize>, 2010 (accessed 11.01.2010.).

[25] G. McKenna, P.F. Allen, D. O'Mahony, A. Flynn, M. Cronin, C. DaMata, N. Woods, Comparison of functionally orientated tooth replacement and removable partial dentures on the nutritional status of partially dentate older patients: a randomised controlled clinical trial, Journal of dentistry 42(6) (2014) 653-9. [26] A. van der Bilt, Assessment of mastication with implications for oral rehabilitation: a review, J Oral Rehabil 38(10) (2011) 754-80.

[27] TheAcademyofProsthodontics, The Glossary of Prosthodontic Terms: Ninth Edition, J Prosthet Dent 117(5S) (2017) e1-e105.

[28] M. Schimmel, P. Christou, F. Herrmann, F. Muller, A two-colour chewing gum test for masticatory efficiency: development of different assessment methods, Journal of oral rehabilitation 34(9) (2007) 671-8.

[29] P. Bourdiol, L. Mioche, Correlations between functional and occlusal toothsurface areas and food texture during natural chewing sequences in humans, Archives of Oral Biology 45(8) (2000) 691-699.

[30] R.S. Manly, C. Pfaffman, D.D. Lathrop, J. Keyser, Oral sensory thresholds of persons with natural and artificial dentitions, J Dent Res 31(3) (1952) 305-12. [31] D.J. Witter, W.H. van Palenstein Helderman, N.H. Creugers, A.F. Kayser, The shortened dental arch concept and its implications for oral health care., Community dentistry and oral epidemiology 27(4) (19999) 249-258. 
[32] D. Armellini, J.A. von Fraunhofer, The shortened dental arch: A review of the literature, Journal of Prosthetic Dentistry 92(6) 531-535.

[33] T. Kanno, G.E. Carlsson, A review of the shortened dental arch concept focusing on the work by the Kayser/Nijmegen group, Journal of oral rehabilitation 33(11) (2006) 850-62.

[34] S. Wolfart, B. Marre, B. Wostmann, M. Kern, T. Mundt, R.G. Luthardt, J. Huppertz, W. Hannak, T. Reiber, N. Passia, G. Heydecke, W. Reinhardt, S. Hartmann, E. Busche, G. Mitov, H. Stark, P. Pospiech, A. Weber, W. Gernet, M.H. Walter, The randomized shortened dental arch study: 5-year maintenance, Journal of dental research 91(7 Suppl) (2012) 65S-71S.

[35] K. Okada, H. Enoki, S. Izawa, A. Iguchi, M. Kuzuya, Association between masticatory performance and anthropometric measurements and nutritional status in the elderly, Geriatr Gerontol Int 10(1) (2010) 56-63.

[36] E. Budtz-Jorgensen, Restoration of the partially edentulous mouth - a comparison of overdentures, removable partial dentures, fixed partial dentures and implant treatment. , Journal of dentistry 24(4) (1996) 237-244.

[37] N.J. Jepson, J.M. Thomason, J.G. Steele, The influence of denture design on patient acceptance of partial dentures, British dental journal 178(8) (1995) 296300.

[38] N.J. Jepson, P.F. Allen, Short and sticky options in the treatment of the partially dentate patient, British dental journal 187(12) (1999) 646-52.

[39] G. McKenna, P.F. Allen, A. Flynn, D. O'Mahony, C. DaMata, M. Cronin, N. Woods, Impact of tooth replacement strategies on the nutritional status of partially-dentate elders, Gerodontology 29(2) (2012) e883-90.

[40] J.S. Kern, S. Wolfart, R.D. Hilgers, B. Marre, H. Scheller, J. Strub, K. Boning, W. Hannak, R.G. Luthardt, G. Heydecke, J. Huppertz, P. Pospiech, B. Wostmann, H. Stark, T. Mundt, F. Jahn, M. Kern, D. Edelhoff, M.H. Walter, The randomized shortened dental arch study: influence of two different treatments on interdental spacing over 5 years, Clin Oral Investig 21(6) (2017) 1945-1951.

[41] M.Z. Nassani, B. Tarakji, K. Baroudi, S. Sakka, Reappraisal of the removable partial denture as a treatment option for the shortened dental arch, European Journal of Dentistry 7(2) (2013) 251-256.

[42] A.F. Kayser, Shortened dental arches and oral function, Journal of oral rehabilitation 8(5) (1981) 457-62.

[43] H. Guiney, H. Woods, H. Whelton, K. Morgan, Predictors of utilisation of dental care services in a nationally

representative sample of adults, Community Dental Health (2011).

[44] K.J. Joshipura, E.B. Rimm, C.W. Douglass, D. Trichopoulos, A. Ascherio, W.C. Willett, Poor oral health and coronary heart disease, Journal of dental research 75(9) (1996) 1631-6.

[45] J.G. Steele, A. Sheiham, W. Marcenes, A.W.G. Walls, Diet and Nutrition in Great Britain, Gerodontology 15(2) (1998) 99-106.

[46] E.I. Flores-Orozco, B. Rovira-Lastra, E. Willaert, M. Peraire, J. MartinezGomis, Relationship between jaw movement and masticatory performance in adults with natural dentition, Acta Odontologica Scandinavica 74(2) (2016) 103107. 